\title{
Impact of Lurasidone and Other Antipsychotics on Body Weight: Real-World, Retrospective, Comparative Study of 15,323 Adults with Schizophrenia
}

\author{
Ilena Pochiero' \\ Fabrizio Calisti ${ }^{1}$ \\ Alessandro Comandini ${ }^{1}$ \\ Alessandra Del Vecchio' \\ Isabella Costamagna' \\ Maria Teresa Rosignoli' \\ Agnese Cattaneo' \\ Sasikiran Nunna ${ }^{2}$ \\ Ilaria Peduto ${ }^{3}$ \\ Franca Heiman ${ }^{3}$ \\ Hsiu-Ching Chang ${ }^{2}$ \\ Chi-Chang Chen (iD) ${ }^{2}$ \\ Christoph Correll ${ }^{4-6}$ \\ 'Angelini Regulatory, Research \& \\ Development, Angelini Pharma S.p.A., \\ Rome, Italy; ${ }^{2} \mathrm{QQVIA}$, Plymouth Meeting, \\ PA, USA; ${ }^{3}$ QQVIA Solutions Italy S.r.l., \\ RWS, Milan, Italy; ${ }^{4}$ Department of Child \\ and Adolescent Psychiatry, Charité \\ Universitätsmedizin, Berlin, Germany; \\ ${ }^{5}$ Department of Psychiatry, The Zucker \\ Hillside Hospital, Northwell Health, Glen \\ Oaks, NY, USA; ${ }^{6}$ Department of \\ Psychiatry and Molecular Medicine, \\ Donald and Barbara Zucker School of \\ Medicine at Hofstra/Northwell, \\ Hempstead, NY, USA
}

Correspondence: Ilena Pochiero Angelini Pharma S.p.A., Viale Amelia 70, Rome, Italy

Email ilena.pochiero@angelinipharma. com
Purpose: The primary objectives were to describe weight changes following initiation of lurasidone versus other antipsychotics and estimate the risk of clinically relevant $(\geq 7 \%)$ weight changes.

Patients and Methods: This retrospective, longitudinal comparative cohort study was based on electronic medical records (EMRs) of United States (US) adult patients with schizophrenia who were prescribed lurasidone or other antipsychotics as monotherapy between 1 April 2013 and 30 June 2019.

Results: Overall, the study included 15,323 patients with a diagnosis of schizophrenia; $6.1 \%$ of patients received lurasidone, $60.4 \%$ received antipsychotics associated with a mediumhigh risk of weight gain (clozapine, olanzapine, quetiapine, risperidone, paliperidone) and $33.5 \%$ received antipsychotics with a low risk of weight gain (aripiprazole, first-generation antipsychotics, ziprasidone). Lurasidone was associated with the smallest proportion of patients experiencing clinically relevant weight gain and the greatest proportion of patients with clinically relevant weight loss. The risk of clinically relevant weight gain was numerically higher with all antipsychotics versus lurasidone and was statistically significant for olanzapine (hazard ratio $[\mathrm{HR}]=1.541 ; 95 \%$ confidence interval $[\mathrm{CI}]=1.121 ; 2.119 ; \mathrm{p}=0.0078$ ) versus lurasidone. The likelihood of $\geq 7 \%$ weight loss was significantly greater with lurasidone versus all antipsychotics $(\mathrm{p}<0.05)$, except ziprasidone.

Conclusion: This real-world study suggests that lurasidone has a lower risk of clinically relevant weight gain and a higher likelihood of clinically relevant weight loss than other commonly used antipsychotics.

Keywords: retrospective studies, weight gain, real world clinical trials

\section{Introduction}

Schizophrenia is a severe and chronic mental illness. ${ }^{1}$ According to current estimates, schizophrenia affects more than 20 million people worldwide. ${ }^{2}$ The onset of schizophrenia is usually between late adolescence and the third decade of life and can profoundly impair education and employment opportunities in affected individuals. ${ }^{3}$ The burden of schizophrenia is considerable and growing worldwide, as highlighted by the Global Burden of Disease Study 2017, which showed a 38.6\% increase in the number of years lived with disability due to schizophrenia from 1990 to 2007 , and a $17.2 \%$ increase from 2007 to $2017 .^{4}$ 
Studies have consistently reported two- to three-fold higher mortality rates among patients with severe and persistent mental illness, including schizophrenia, compared with the general population, which translates into a reduction in life expectancy of 10-20 years. ${ }^{5-7}$ Cardiovascular disease (CVD) is a major contributor to the premature death of patients with serious mental disorders. ${ }^{5,7}$ A recent large-scale meta-analysis investigating CVD prevalence, incidence and related mortality in people with severe mental illness estimated that, after adjustment for several confounders, affected individuals have a $78 \%$ higher risk of developing CVD and an $85 \%$ increased risk of dying from CVD than the control population. ${ }^{5}$ This meta-analysis also found that the incidence of CVD was significantly increased with the use of antipsychotics, higher body mass index (BMI) and higher baseline prevalence of CVD. ${ }^{5}$ Studies have also shown that individuals with severe mental illness have a significantly higher risk of developing metabolic syndrome, an established predictor of CVD, than the general population. ${ }^{7,8}$ Furthermore, overweight and obesity are common conditions among patients with schizophrenia. ${ }^{9,10}$ Besides affecting health-related quality of life, overweight and obesity are well-established and modifiable risk factors of CVD. ${ }^{9}$

Antipsychotics constitute the standard treatment for patients with schizophrenia. ${ }^{3}$ Originally, they were classified according to their chemical structure. First-generation (or typical) antipsychotics classification is related to the prevailing sedative or antipsychotic potential, while second-generation (or atypical) antipsychotics classification is mainly related to their pharmacodynamic properties. ${ }^{11}$ Although the available antipsychotics appear to have comparable efficacy with regard to symptom improvement, their adverse effect profiles can differ significantly. ${ }^{12}$ First-generation (or typical) antipsychotics are mainly associated with extrapyramidal motor effects and elevated prolactin levels, while second-generation (or atypical) antipsychotics can induce varying degrees of weight gain, metabolic abnormalities and sedation. ${ }^{8,10,12-}$ 14 Aside from contributing to CVD, weight gain can adversely affect quality of life ${ }^{15,16}$ and is one of the most frequent reasons leading to treatment non-adherence or discontinuation among patients with schizophrenia. ${ }^{17,18}$ The reason why atypical antipsychotics are more likely associated to weight gain and metabolic syndrome is still not clear. Among the possible reasons, there are factors such as genetic polymorphisms and the interaction with neurotransmitter receptors. ${ }^{19}$

Despite the introduction of second-generation antipsychotics, the pharmaceutical management of schizophrenia is still a clinical challenge. ${ }^{20}$ Combination therapy is often required for patients who do not respond to the therapy. However, adding a second antipsychotic medication might be associated with potential risks that have not been studied in prospective trials. ${ }^{21}$

Lurasidone is a second-generation (or atypical) antipsychotic approved in 2010 by the US Food and Drug Administration for the treatment of schizophrenia and bipolar depression in adults and adolescents, ${ }^{22}$ and in 2014 by the European Medicines Agency for the treatment of schizophrenia in adults. ${ }^{23}$ In 2020, lurasidone was the first atypical antipsychotic to be approved in Europe for the treatment of schizophrenia in adolescents aged 13-17 years. ${ }^{24}$ In adults, the antipsychotic efficacy and safety of lurasidone were investigated through an extensive clinical trial program, including 6-week, placebo- and active-controlled trials, and long-term, open-label maintenance studies. ${ }^{25-28}$ These trials highlighted the limited impact of lurasidone on weight and metabolic parameters. For example, in the Phase 3 trial by Meltzer et al, $5.9 \%$ of patients who received lurasidone (40 or $120 \mathrm{mg} /$ day) had a clinically relevant $(\geq 7 \%)$ weight increase compared with $34.4 \%$ and $7.0 \%$ of those receiving olanzapine and placebo, respectively. ${ }^{27}$ A post-hoc analysis of the pooled data from six randomized controlled trials with lurasidone (40-160 mg/day) showed that lurasidone was associated with a mean change in weight of $-0.4 \mathrm{~kg}$ at 12 months, while the active comparators were associated with weight increases of $+2.6 \mathrm{~kg}$ for risperidone and $+1.2 \mathrm{~kg}$ for quetiapine extended release (XR). ${ }^{29}$

However, real-world evidence of the effectiveness of lurasidone and its impact on body weight in schizophrenia patients managed in routine clinical practice remains limited. Here, we present a study based on electronic medical records (EMRs) of patients with schizophrenia who were prescribed antipsychotics, including lurasidone, between 2013 and 2019, in an ambulatory care setting in the US (IQVIA US ambulatory database). The study was prompted by the need for real-world evidence of the effects of lurasidone monotherapy on body weight in adults with schizophrenia. The primary objectives were to 1) report on a real-world population of adults with schizophrenia receiving antipsychotic treatment in routine clinical practice; 2) describe changes 
in body weight during treatment with lurasidone versus other antipsychotics; and 3) estimate the risk of clinically relevant weight changes associated with lurasidone versus other antipsychotics.

\section{Patients and Methods Study Design}

This retrospective, longitudinal comparative cohort study was based on EMRs of US adults with schizophrenia who were prescribed lurasidone or other antipsychotics as monotherapy between 1 April 2013 and 30 June 2019. Patient data covered a period extending from 1 April 2012 to 31 August 2019 as the eligibility for the analysis required the availability of data over at least 12 months preceding the date of the first prescription of antipsychotic (baseline period) and at least 2 months or up to 12 months following the date of the first antipsychotic prescription (follow-up period).

\section{Data Source}

The data analysed in this study were extracted from the IQVIA US Ambulatory EMR database. This database contains approximately 68 million patient records obtained from a network of healthcare providers, comprising 100,000 physicians (50\% primary care practitioners and $50 \%$ specialists) from approximately 800 large practices and physician networks. Records from the IQVIA US Ambulatory EMR database have been available since 2001 and are continuously updated. The information collected in this EMR database includes patient demographics, patient vital signs (blood pressure, heart rate, body weight and height), CVD risk factors (smoking and alcohol consumption), psychiatric and medical comorbidities, results from performed laboratory tests, allergies and vaccinations, psychiatric diagnoses (International Classification of Diseases, Ninth Revision, Clinical Modification [ICD-9-CM] and ICD-10-CM), drugs prescribed with dosage information, procedures performed and other treatments (Current Procedural Terminology and Healthcare Common Procedure Coding System), and medical care received. This information is valuable for identifying possible associations between patient characteristics and clinical variables and diagnosis, prescribed treatments, and therapeutic outcomes in the setting of real-world clinical practice.

\section{Patients}

The study population included adult patients (aged $\geq 18$ years) with schizophrenia, identified using the diagnosis codes 295.XX (ICD-9-CM) or F20.X (ICD-10-CM).

To be eligible for this analysis, each patient was required to meet all inclusion criteria and none of the exclusion criteria. The inclusion criteria were: i) diagnosis of schizophrenia during the study period (1 April 2012 to 31 August 2019); ii) male and female patients aged $\geq 18$ years at index date (ie, the date of first prescription of the antipsychotic); iii) at least one prescription of antipsychotic as monotherapy between 1 April 2013 and 30 June 2019; iv) available data for at least 12 months before the index date (baseline period) and for at least 2 months or up to 12 months after the index date (follow-up period); v) at least one measure of weight or BMI recorded during the 12-month baseline period; and vi) at least one measure of weight or BMI recorded between 1 month after the index date and the end of the follow-up period. The exclusion criteria were: i) prescription of more than one antipsychotic at the index date; and ii) prescription of any antipsychotic during the baseline period.

For analysis purposes, eligible patients were subdivided into three cohorts - cohorts $\mathrm{A}, \mathrm{B}$ or $\mathrm{C}$ - based on the antipsychotic prescribed at the index date. Cohort A comprised patients prescribed lurasidone; cohort $\mathrm{B}$ and cohort $\mathrm{C}$ comprised patients prescribed treatment with antipsychotics associated with a low- and a medium-high risk for weight gain, respectively. Ranking of the risk of weight gain associated with antipsychotics was based on published evidence at the time of study design ${ }^{30-32}$. According to this evidence, commonly used low-risk antipsychotics in terms of weight gain included aripiprazole, ziprasidone and high-potency first-generation antipsychotics (haloperidol), and commonly used medium-high risk antipsychotics included clozapine, olanzapine, quetiapine, risperidone and paliperidone.

\section{Study Variables and Outcomes}

The variables extracted from the IQVIA US Ambulatory EMR database included demographic characteristics (age, sex, ethnicity and region of residence), weight, BMI and metabolic parameters (waist circumference or BMI, blood pressure, fasting glucose, serum triglycerides, serum highdensity lipoprotein cholesterol, serum low-density lipoprotein cholesterol and glycated haemoglobin). Duration of antipsychotic exposure, defined as the time from the index 
date to the end of treatment or follow-up, was also extracted. The presence, or absence, of comorbidities of interest during the 12-month baseline period was also extracted. These comorbidities were identified by their ICD-9-CM or ICD-10-CM codes, and included major depressive disorder, anxiety disorders, substance abuse, arterial hypertension, hyperlipidaemia, diabetes, hyperprolactinaemia and insomnia.

Data were extracted according to the following time periods: over 12 months before the index date for the baseline data; and for a minimum of 2 months and a maximum of 12 months from the index date for the follow-up data, unless the patient was switched off lurasidone (cohort A) or switched to an antipsychotic or having added a second antipsychotic belonging to a different class of risk for weight gain (cohorts B and C). In this case, the follow-up ended when the new antipsychotic was prescribed, or all antipsychotics were stopped.

The primary objectives of the study were to 1) characterise a real-world schizophrenia population, after stratification by antipsychotic prescribed at index date; 2) describe weight changes following the initiation of antipsychotic treatment at index date (lurasidone vs other low or medium/high weight gain risk antipsychotics); and 3) estimate the risk of clinically relevant weight changes associated with lurasidone versus other low or medium/ high weight gain risk antipsychotics.

A change in weight following the initiation of antipsychotic treatment was considered clinically relevant when weight increased or decreased by at least 7\% (the usual threshold used in randomised clinical trials to define excessive weight gain), ${ }^{33}$ or when a shift in BMI category occurred (eg, from "overweight" to "obese" or vice versa), based on the measure taken at index date or within 1 month before the index date. When several weight or BMI measures were available in the follow-up period, the lowest value was considered.

\section{Statistical Analysis}

Categorical variables were reported using percentages, while continuous variables were summarised as mean (standard deviation [SD]) or median (range) values. All variables were stratified by the antipsychotic prescribed at the index date. Differences in the variables between lurasidone (cohort A) and the antipsychotics of cohorts B and $\mathrm{C}$, as well as individual antipsychotics of interest (aripiprazole, ziprasidone and haloperidol from cohort $\mathrm{B}$, and olanzapine, quetiapine and risperidone from cohort $\mathrm{C}$ ) were tested using the chi-squared test and the Fisher's exact test. Times to clinically relevant weight and BMI changes following antipsychotic treatment were estimated using Kaplan-Meier curves; the univariate comparison of lurasidone versus cohorts $\mathrm{B}$ or $\mathrm{C}$ or individual antipsychotics was performed using the Log rank test with Bonferroni adjustments. The likelihood of clinically relevant changes in weight associated with antipsychotic treatment and potentially confounding factors (ie, age, sex, region of residence, ethnicity, comorbidities, index year and weight at index date or within 1 month before the index date) was estimated using a multivariate Cox Proportional Hazards model. All statistical analyses were performed using the $\mathrm{SAS}^{\circledR}$ software, version 9.4.

\section{Results}

\section{Study Population}

The IQVIA US EMR database analysis identified 122,464 patients with a diagnosis of schizophrenia between April 2012 and August 2019. Among the 122,464 identified patients, 15,323 (12.5\%) met all inclusion criteria and did not have an antipsychotic prescription during the baseline period or a prescription of more than one antipsychotic at the index date. Figure 1 describes the details of the patient selection procedure that resulted in the final sample of 15,323 patients, who were eligible for this retrospective analysis.

Between April 2013 and June 2019, the majority of patients $(9250 / 15,323 ; 60.4 \%)$ were prescribed an antipsychotic associated with a medium-high risk of inducing weight increase (cohort C), and 5138 patients (33.5\%) were prescribed a low-risk antipsychotic (cohort B). Lurasidone was prescribed to a minority of patients (935/ 15,$323 ; 6.1 \%$ [cohort A]). When the antipsychotics were analysed individually, the most frequently prescribed were risperidone $(20.4 \%)$, quetiapine $(18.2 \%)$ and olanzapine (14.1\%), all three were associated with a medium-high risk of weight gain. The mean (SD) antipsychotic exposure duration in the group prescribed lurasidone was 70.1 (75.9) days; the mean antipsychotic exposure in cohorts B and C was 77.2 days (83.6) and 84.6 (90.5) days, respectively, with differences between lurasidone and the other cohorts being statistically significant $(p<0.05)$.

Demographic characteristics and comorbidities of the entire population, as well as of patients stratified according to antipsychotics prescribed at the index date, are summarized in Table 1. Female and male patients were 
Patients diagnosed with schizophrenia ${ }^{a}$ during study period

(1 Apr 2012 to 31 Aug 2019)

$$
(\mathrm{N}=122,464)
$$

Patients with $\geq 1 \mathrm{AP}$ prescribed as monotherapy during selection period

(1 April 2013 to 30 June 2019)

$$
(n=84,870)
$$

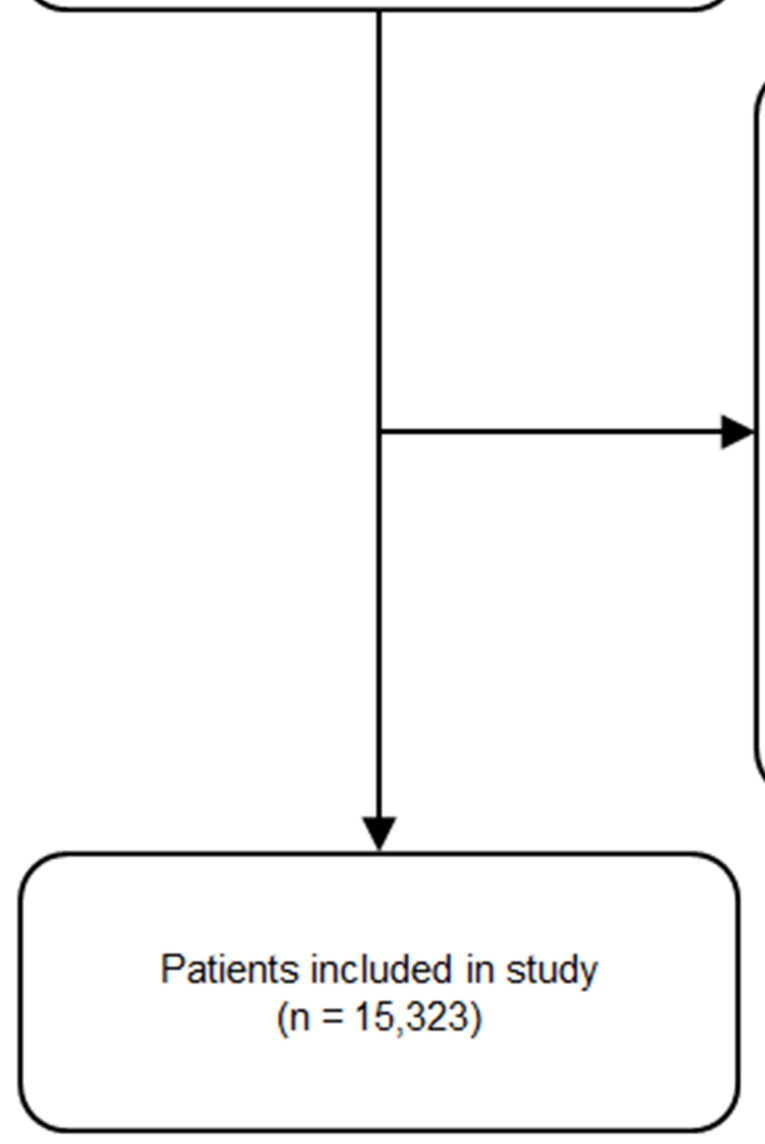

эCD-9-CM diagnosis 295.XX or ICD-10-CM diagnosis F20.XX.

bPatients could be excluded for more than one reason. Excluded patients were reported in the order in which the exclusion criteria where applied in the database

cDefined as 12 months before index date.

DDefined as $\geq 2$ months or up to 12 months after index date.

Figure I Patient selection process from IQVIA US electronic medical records database.

Abbreviations: AP, antipsychotic; BMI, body mass index; ICD, International Classification of Diseases; CM, Clinical Modification. 


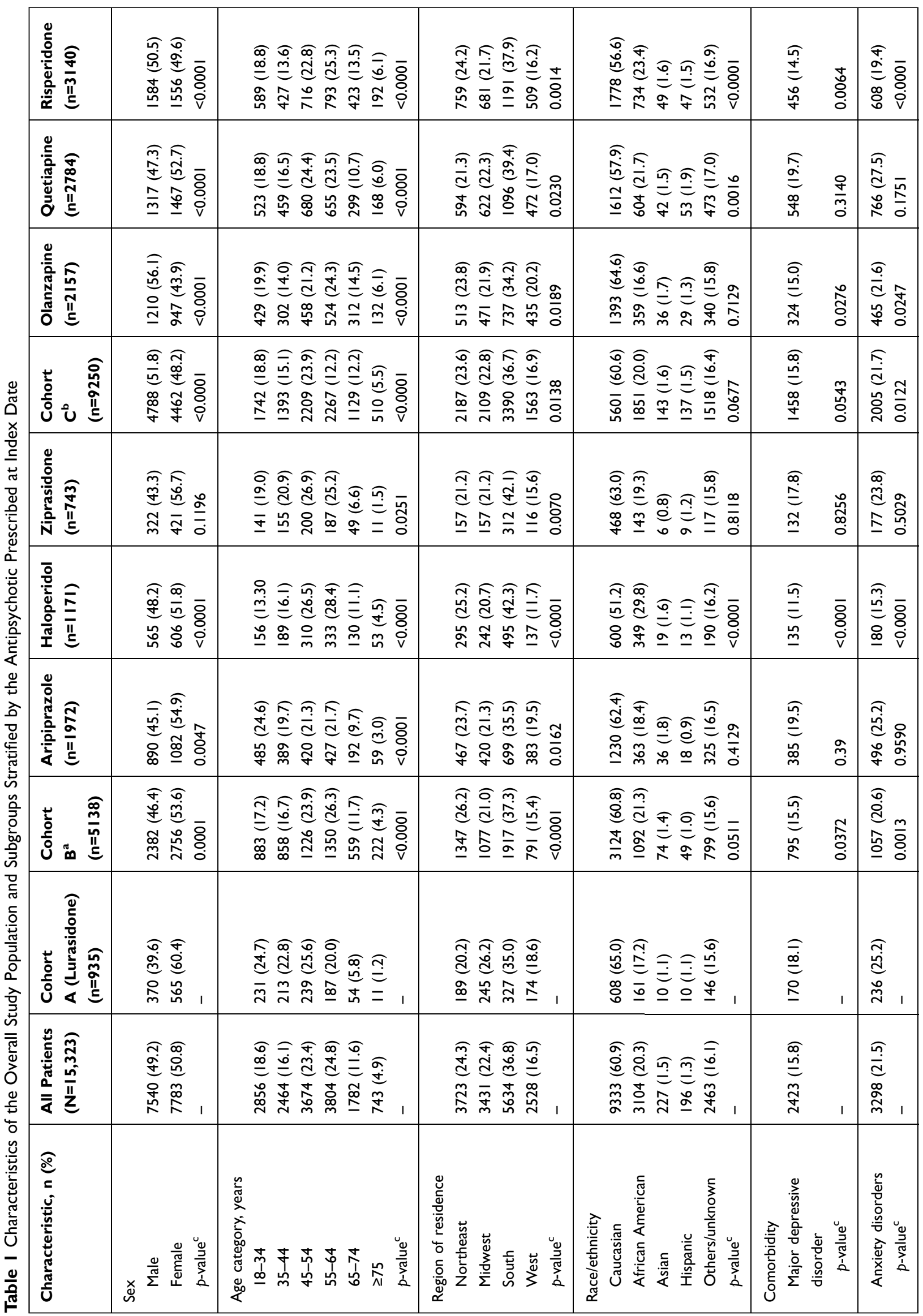




\begin{tabular}{|c|c|c|c|c|c|}
\hline 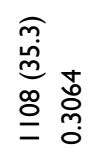 & 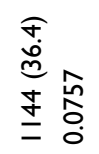 & 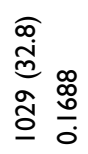 & 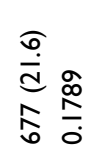 & 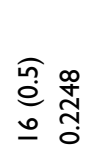 & 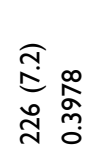 \\
\hline 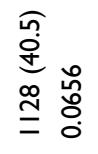 & $\begin{array}{l}\widehat{0} \\
\hat{n} \\
\hat{0} \\
\text { 产 } \\
\underline{0}\end{array}$ & 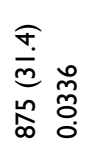 & 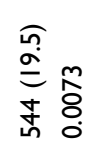 & $\underset{\substack{\tilde{e} \\
\stackrel{e}{e}}}{ }$ & 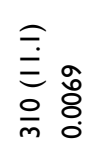 \\
\hline 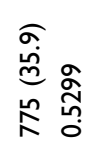 & 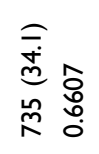 & 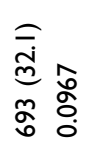 & 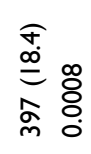 & $\stackrel{\widehat{m}}{\stackrel{\rho}{n}}, 1$ & 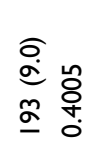 \\
\hline 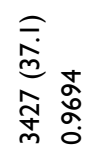 & 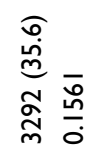 & 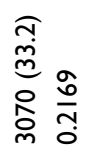 & 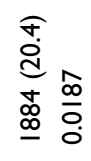 & 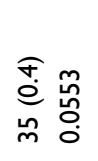 & 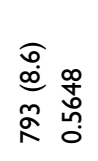 \\
\hline 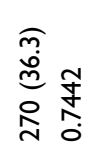 & 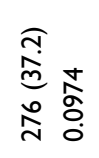 & 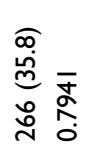 & 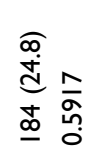 & $\stackrel{\widehat{m}}{\stackrel{0}{c}}$, & 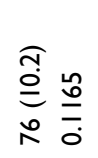 \\
\hline 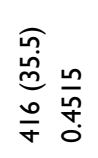 & 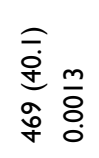 & 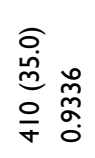 & 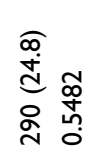 & $\stackrel{\widehat{m}}{\stackrel{0}{\rho}}$ & 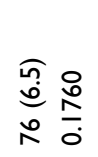 \\
\hline 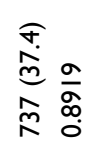 & 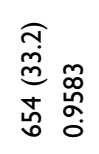 & 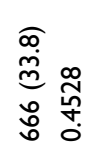 & 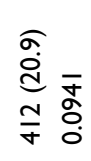 & 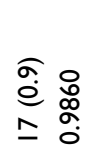 & 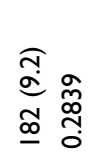 \\
\hline 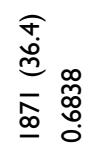 & 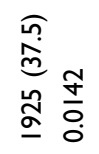 & 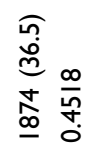 & 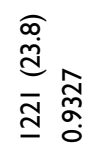 & 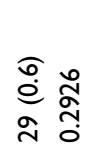 & 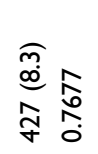 \\
\hline 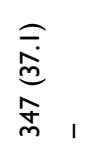 & 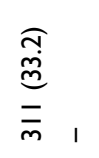 & 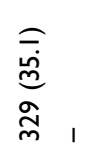 & $\begin{array}{l}\widehat{\widehat{D}} \\
\stackrel{d}{d} \\
\bar{d}\end{array}$ & $\begin{array}{l}\hat{\sigma} \\
\stackrel{\rho}{0} \\
\infty\end{array}$ & $\begin{array}{l}\widehat{\sigma} \\
\infty \\
\stackrel{\infty}{n} \\
\stackrel{n}{n}\end{array}$ \\
\hline 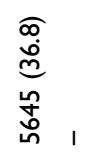 & $\begin{array}{l}\widehat{\overline{\dot{0}}} \\
\stackrel{\infty}{\infty} \\
\stackrel{0}{0} \\
\hat{n}\end{array}$ & 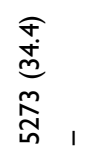 & 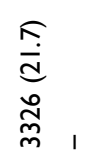 & 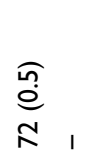 & $\begin{array}{l}\tilde{\Omega} \\
\infty \\
\infty \\
\stackrel{\alpha}{\Omega}\end{array}$ \\
\hline 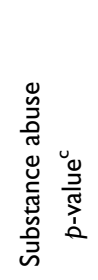 & 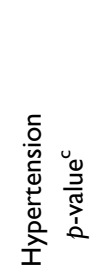 & 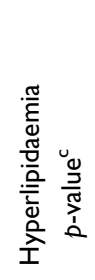 & 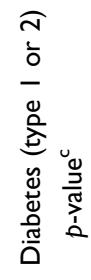 & 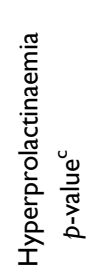 & 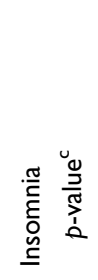 \\
\hline
\end{tabular}

equally represented in the overall population (50.8\% females, $49.2 \%$ males) and in most treatment subgroups, except for the cohort treated with lurasidone, which showed a significantly higher proportion of female patients (60.4\%) than cohort B (53.6\%) and cohort C $(48.2 \%)$. In the entire study population, the most frequently represented age group was 45-64 years, accounting for nearly $50 \%$ of the sample; the youngest age subgroup (18-34 years) accounted for $18.6 \%$ of the study population. Overall and across all subgroups, the most frequently reported regions of residence were Southern states (overall, 36.8\%) and the majority of patients were of white ethnicity (overall, 60.9\%). During the 12-month baseline period preceding the initiation of antipsychotic treatment, the most common comorbidities of interest were substance abuse (36.8\%), hypertension (36.1\%) and hyperlipidaemia (34.4\%). Anxiety disorders and major depressive disorder were slightly more prevalent in patients prescribed lurasidone $(25.2 \%$ and 18.1 , respectively) than in cohort B $(20.6 \%$ and $15.5 \%$, respectively) and cohort $\mathrm{C}(21.7 \%$ and $15.8 \%$, respectively; $p \leq$ 0.05 for all lurasidone vs cohort $\mathrm{B}$ and $\mathrm{C}$ comparisons). However, no major differences were detected overall in the comorbidity profile across treatment cohorts and subgroups.

\section{Changes in Weight and BMI}

Weight measures recorded at index date and during follow-up for the overall population, as well as subgroups stratified according to the antipsychotic prescribed, are summarized in Table 2. At index date, the mean $\pm \mathrm{SD}$ weight of the study population was $89.6 \pm 24.2 \mathrm{~kg}$. Patients treated with lurasidone had a higher mean weight $(94.7 \pm 26.2 \mathrm{~kg})$ than the overall study sample, cohort B $(91.6 \pm 24.7 \mathrm{~kg} ; p=0.0013)$ and cohort C $(87.9 \pm$ $23.5 \mathrm{~kg}: p<0.0001$ ), and compared with most individual antipsychotics. Before initiating treatment, the lurasidone cohort included a significantly greater proportion of obese patients (BMI $\geq 30 \mathrm{~kg} / \mathrm{m}^{2}$ ) compared with other cohorts. Mean \pm SD weights recorded during the follow-up period overlapped with those of the baseline period: $89.9 \pm$ $23.9 \mathrm{~kg}$ for the overall population, $94.9 \pm 25.8 \mathrm{~kg}$ for lurasidone, $91.7 \pm 24.4 \mathrm{~kg}$ for cohort $\mathrm{B}$ and $88.4 \pm$ $23.3 \mathrm{~kg}$ for cohort C. Univariate differences between lurasidone and cohorts $\mathrm{B}$ and $\mathrm{C}$ were statistically significant ( $p<0.001$ for both comparisons).

In the analysis of clinically relevant weight changes $(\geq 7 \%)$ from baseline to follow-up after treatment 


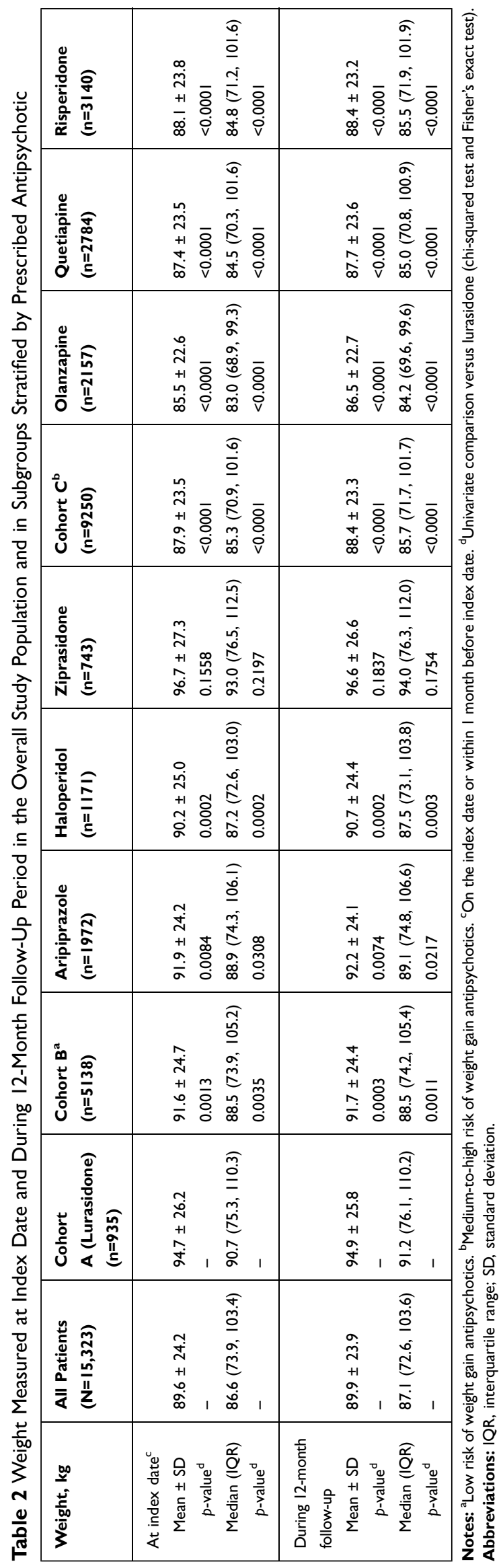

initiation, the proportion of patients experiencing clinically relevant weight gain was numerically smallest with lurasidone and numerically greatest with olanzapine (Figure 2). Overall, 9.4\% of the study population experienced a clinically relevant weight gain; this proportion was $7.7 \%$ in the lurasidone cohort, $8.6 \%$ in cohort B and $10.2 \%$ in cohort $\mathrm{C}$. The proportion of patients with clinically relevant weight loss was greatest with lurasidone (21.8\%); this proportion was $19.0 \%$ in the overall population, $18.0 \%$ in cohort $\mathrm{B}$ and $18.9 \%$ in cohort C. Aripiprazole was associated with the smallest proportion of patients (17.2\%) with clinically relevant weight loss (Figure 2).

In a comparison of lurasidone with other individual antipsychotics by Kaplan-Meier analysis, the time to first clinically relevant weight change was significantly longer with lurasidone only when compared with olanzapine $(p=$ 0.0012 ). With regard to a $\geq 7 \%$ weight loss, the time to this event was significantly shorter with lurasidone than aripiprazole $(p=0.0017)$, while the other comparisons did not reach statistical significance. The analysis of clinically relevant weight changes as defined by a shift in BMI category provided similar results. Lurasidone-treated patients showed a trend towards longer times to the first shift to a higher category (worsening) and shorter times to the first shift to a lower category (improvement) compared with all other antipsychotics. Statistical significance was reached for the lurasidone versus olanzapine comparison ( $p=0.0118)$, with lurasidone being associated with a significantly longer time to BMI category worsening.

Tables 3 and 4 summarize the multivariate Cox proportional hazards analysis of the risk of a clinically relevant weight change during treatment with lurasidone versus other antipsychotics, with adjustment for key demographic and clinical characteristics. The risk of clinically relevant weight gain was numerically greater with all antipsychotics compared with lurasidone. However, statistical significance was only reached for the comparisons between olanzapine and lurasidone (hazard ratio [HR] 1.541; 95\% CI 1.121-2.119; $p=0.0078$ ); Table 3). The risk of clinically relevant weight gain was also significantly greater for young patients (aged 18-34 years) and for patients who initiated treatment more recently, while the presence of hyperlipidaemia in the baseline period and a lower weight at index period predicted a significantly lower risk of clinically relevant weight gain with antipsychotic treatment. Lurasidone was associated with a significantly 


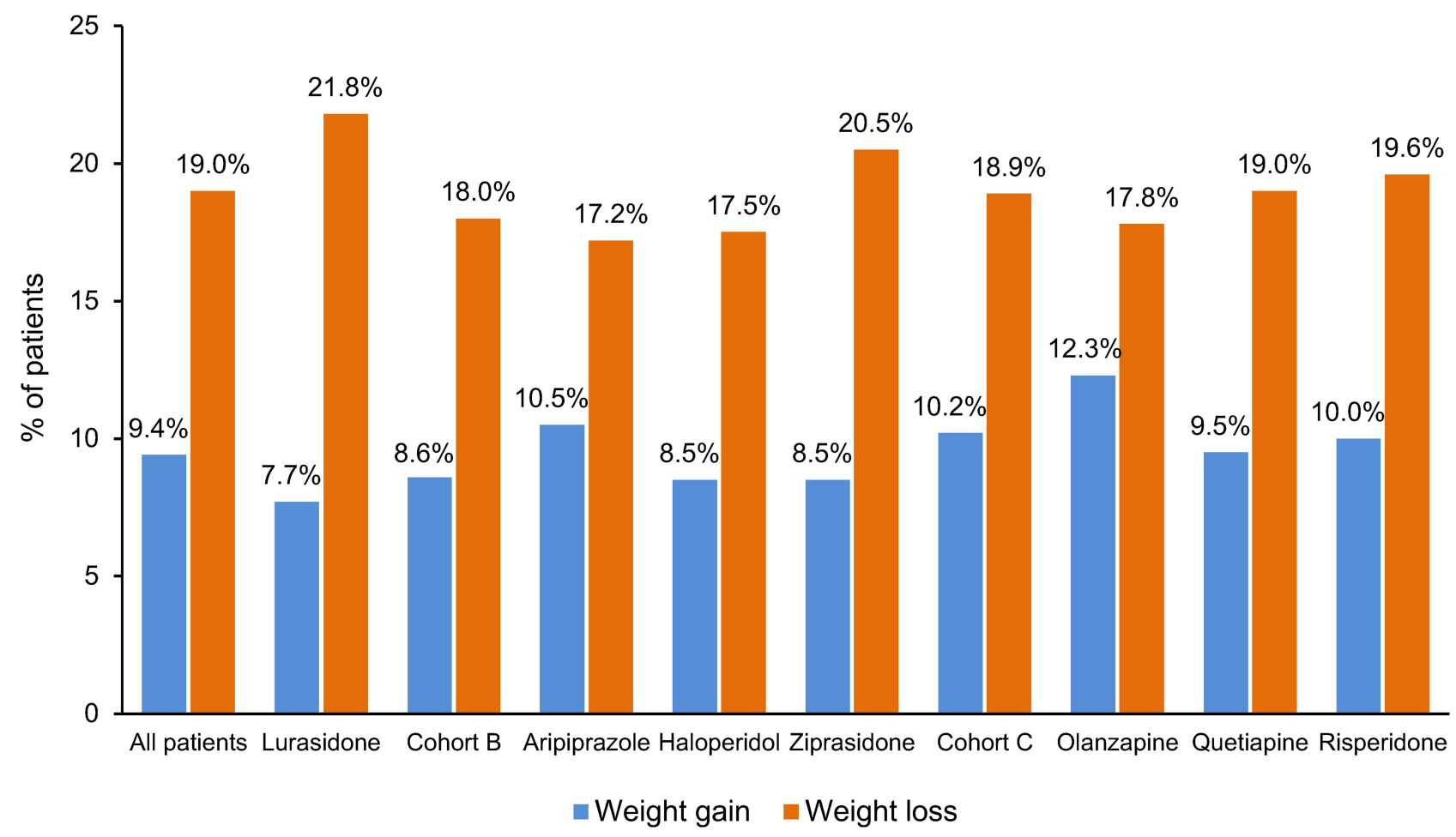

Figure 2 Proportion of patients showing clinically relevant changes in weight (gain or loss) during treatment with the antipsychotic prescribed at the index date.

greater likelihood of clinically relevant weight loss than other antipsychotics, except ziprasidone (Table 4).

When clinically relevant weight changes were defined as a shift in BMI category, risk estimates provided similar results (see Supplementary Tables S1 and S2). Lurasidone was associated with a lower risk of a shift to a higher BMI category than all other antipsychotics, with statistical significance being reached for comparisons with aripiprazole, olanzapine and quetiapine. The likelihood of a shift to a lower BMI category was greater with lurasidone versus all other antipsychotics, with statistical significance being reached for comparisons with aripiprazole. Younger age (18-34 years) was a significant predictor of BMI worsening.

\section{Discussion}

This retrospective analysis of schizophrenia patients from the IQVIA US Ambulatory EMR database investigated the issue of weight and BMI changes associated with antipsychotic treatment. The main objective of this analysis was to determine whether the minimal effect of lurasidone on weight observed in randomised clinical trials could be confirmed in patients managed in routine clinical practice.

The cohort of patients with schizophrenia identified from the IQVIA database was representative of mi patients with severe mental illness encountered in real-world clinical practice in the US. More than half of the cohort were aged between 45 and 65 years and, in line with published evidence, ${ }^{5,7,9}$ substantial proportions of the population presented with comorbidities that constitute well-established CVD risk factors, including hypertension, hyperlipidaemia, and obesity. Notably, $60 \%$ of patients were prescribed second-generation antipsychotics known to have a medium-high risk of causing weight gain, while lurasidone was prescribed to less than $10 \%$ of patients.

The results of the present analysis suggest that in the complex setting of real-world management of schizophrenia, lurasidone is associated with less clinically relevant weight gain and more clinically relevant weight loss than other antipsychotics, consistent with the findings of previous clinical trials. ${ }^{27,29,34}$ Compared with the other antipsychotics, lurasidone was associated with the smallest proportion of patients with clinically relevant weight gain and with the greatest proportion of patients with clinically relevant weight loss, although not all the comparison reached statistical significance. It is worth noting that compared to aripiprazole, the likelihood of experiencing a clinically relevant weight loss was significantly higher for lurasidone. However, lurasidone was not associated with a statistically lower likelihood of clinical meaningful 
Table 3 Multivariate Cox Proportional Hazards Model Analysis of the Risk of Clinically Relevant ( $\geq 7 \%)$ Weight Gain

\begin{tabular}{|c|c|c|}
\hline Parameter & HR $(95 \% \mathrm{Cl})$ & p-value ${ }^{a}$ \\
\hline \multicolumn{3}{|l|}{ Antipsychotic } \\
\hline \multicolumn{3}{|l|}{ Lurasidone (REF) } \\
\hline Aripiprazole (SGA) & $1.133(0.814 ; 1.577)$ & 0.4589 \\
\hline Ziprasidone (SGA) & I.I $27(0.738 ; 1.72 \mathrm{I})$ & 0.5810 \\
\hline Haloperidol (FGA) & $1.220(0.832 ; 1.789)$ & 0.3082 \\
\hline Olanzapine (SGA) & $1.541(1.121 ; 2.119)$ & 0.0078 \\
\hline Quetiapine (SGA) & $0.900(0.649 ; 1.248)$ & 0.5269 \\
\hline Risperidone (SGA) & $1.226(0.895 ; 1.680)$ & 0.2040 \\
\hline $\begin{array}{l}\text { Duration of index } \\
\text { antipsychotic }\end{array}$ & 0.999 (0.999; 1.000$)$ & 0.2433 \\
\hline \multicolumn{3}{|l|}{ Index year } \\
\hline \multicolumn{3}{|l|}{2013 (REF) } \\
\hline 2014 & $1.245(0.946 ; 1.637)$ & 0.1173 \\
\hline 2015 & $1.456(1.107 ; 1.915)$ & 0.0072 \\
\hline 2016 & $1.432(1.074 ; 1.911)$ & 0.0146 \\
\hline 2017 & $1.710(1.277 ; 2.291)$ & 0.0003 \\
\hline 2018 & $1.684(1.236 ; 2.293)$ & 0.0010 \\
\hline 2019 & $3.401(2.210 ; 5.234)$ & $<0.0001$ \\
\hline \multicolumn{3}{|l|}{ Sex } \\
\hline \multicolumn{3}{|l|}{ Female (REF) } \\
\hline Male & $1.081(0.933 ; 1.253)$ & 0.2973 \\
\hline \multicolumn{3}{|l|}{ Age group category, years } \\
\hline \multicolumn{3}{|l|}{ 18-34 (REF) } \\
\hline $35-44$ & $0.727(0.592 ; 0.892)$ & 0.0023 \\
\hline $45-54$ & $0.523(0.425 ; 0.644)$ & $<0.0001$ \\
\hline $55-64$ & $0.416(0.333 ; 0.520)$ & $<0.0001$ \\
\hline $65-74$ & $0.304(0.219 ; 0.421)$ & $<0.0001$ \\
\hline$\geq 75$ & $0.264(0.164 ; 0.424)$ & $<0.0001$ \\
\hline \multicolumn{3}{|l|}{ Region of residence } \\
\hline \multicolumn{3}{|l|}{ Northeast (REF) } \\
\hline Midwest & $1.092(0.884 ; 1.347)$ & 0.4142 \\
\hline South & $1.027(0.847 ; 1.246)$ & 0.7840 \\
\hline West & $0.956(0.759 ; 1.203)$ & 0.6997 \\
\hline \multicolumn{3}{|l|}{ Race/ethnicity } \\
\hline \multicolumn{3}{|l|}{ Caucasian (REF) } \\
\hline African American & 0.967 (0.80I; I.I67) & 0.7262 \\
\hline Asian & 0.716 (0.39I; I.3I2) & 0.2800 \\
\hline Hispanic & $1.438(0.893 ; 2.315)$ & 0.1355 \\
\hline Others/unknown & 1.037 (0.858; I.254) & $0.705 I$ \\
\hline \multicolumn{3}{|l|}{ Comorbidity } \\
\hline Major depressive disorder & $1.053(0.869 ; 1.276)$ & 0.5992 \\
\hline Anxiety disorders & $1.102(0.928 ; 1.308)$ & 0.2671 \\
\hline Substance abuse & $1.029(0.887 ; 1.193)$ & 0.7093 \\
\hline Hypertension & $1.013(0.836 ; 1.227)$ & 0.8947 \\
\hline Hyperlipidaemia & $0.727(0.594 ; 0.890)$ & 0.0020 \\
\hline Diabetes (type I or 2) & $0.963(0.764 ; 1.213)$ & 0.7470 \\
\hline
\end{tabular}

(Continued)
Table 3 (Continued)

\begin{tabular}{|l|l|l|}
\hline Parameter & HR (95\% CI) & -value $^{\mathrm{a}}$ \\
\hline \multicolumn{1}{|c|}{ Hyperprolactinaemia } & I.I89 $(0.443 ; 3.192)$ & 0.73 II \\
Insomnia & I.037 $(0.808 ; 1.329)$ & 0.7766 \\
\hline $\begin{array}{l}\text { Weigh at index date or within } \\
\text { I month before index date }\end{array}$ & $0.976(0.973 ; 0.980)$ & $<0.000$ I \\
\hline
\end{tabular}

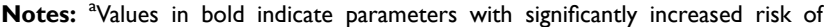
clinically relevant $(\geq 7 \%)$ weight gain.

Abbreviations: $\mathrm{Cl}$, confidence interval; $\mathrm{FGA}$, first-generation antipsychotic; $\mathrm{HR}$, hazard ratio; REF, reference; SGA, second-generation antipsychotic.

weight gain than aripiprazole. This finding appears to be consistent with that from a previous study showing that, among antipsychotics, aripiprazole and lurasidone were among the ones associated with the smallest risk of weight gain. $^{13}$

Current real-world evidence concerning lurasidone use is very limited, and this study is, to our best knowledge, the first to analyse a population of $>15,000$ patients with a diagnosis of schizophrenia. A previous real-world analysis of weight changes associated with lurasidone treatment included 439 patients, $65.8 \%$ of whom had a diagnosis on the schizophrenia-spectrum or bipolar disorder. ${ }^{10}$ The results of this previous real-world analysis of the impact of lurasidone on weight were consistent with those from clinical trials, showing that lurasidone was associated with a mean reduction in weight of $0.77 \mathrm{~kg}$ during a follow-up period of 12 months..$^{10}$ The weight loss was more substantial in patients previously treated with second-generation antipsychotics associated with medium-high risk of weight gain and amounted to $1.68 \mathrm{~kg}$ over 12 months. $^{10}$ In our analysis, more than $20 \%$ of patients treated with lurasidone experienced a clinically relevant weight loss. The proportions of patients treated with other antipsychotics and achieving this result were all numerically smaller than with lurasidone, but substantially higher than those reported in the literature. For example, in a pooled analysis of long-term trials with lurasidone by Meyer et al, the proportion of patients with $\geq 7 \%$ weight loss in the lurasidone group $(18.5 \%)$ was more than twice that in the quetiapine XR (9.1\%) and risperidone $(6.7 \%)$ groups. $^{29}$

Weight gain is increasingly recognised as a relevant outcome in the management of patients with severe mental illness, as this group presents several risk factors for obesity and metabolic alterations, besides the use of antipsychotics, including inadequate medical care, sedentary 
Table 4 Multivariate Cox Proportional Hazards Model Analysis of the Risk of Clinically Relevant ( $\geq 7 \%$ ) Weight Loss

\begin{tabular}{|c|c|c|}
\hline Parameter & HR (95\% Cl) & p-value ${ }^{a}$ \\
\hline \multicolumn{3}{|l|}{ Antipsychotic } \\
\hline \multicolumn{3}{|l|}{ Lurasidone (REF) } \\
\hline Aripiprazole (SGA) & 0.647 (0.52I; 0.802) & $<0.0001$ \\
\hline Ziprasidone (SGA) & $0.949(0.741 ; 1.216)$ & 0.6815 \\
\hline Haloperidol (FGA) & $0.756(0.593 ; 0.965)$ & 0.0246 \\
\hline Olanzapine (SGA) & $0.723(0.585 ; 0.894)$ & 0.0027 \\
\hline Quetiapine (SGA) & $0.792(0.649 ; 0.966)$ & 0.0212 \\
\hline Risperidone (SGA) & $0.78 \mathrm{I}(0.642 ; 0.95 \mathrm{I})$ & 0.0137 \\
\hline $\begin{array}{l}\text { Duration of index } \\
\text { antipsychotic }\end{array}$ & $1.001(1 ; 1.001)$ & 0.0417 \\
\hline \multicolumn{3}{|l|}{ Index year } \\
\hline \multicolumn{3}{|l|}{2013 (REF) } \\
\hline 2014 & $0.930(0.793 ; 1.091)$ & 0.3732 \\
\hline 2015 & $0.894(0.756 ; 1.056)$ & 0.1872 \\
\hline 2016 & $0.77(0.639 ; 0.927)$ & 0.0058 \\
\hline 2017 & $0.849(0.699 ; 1.031)$ & 0.0984 \\
\hline 2018 & $0.932(0.76 I ; 1.14 I)$ & 0.4955 \\
\hline 2019 & $2.328(1.626 ; 3.332)$ & $<0.0001$ \\
\hline \multicolumn{3}{|l|}{ Sex } \\
\hline \multicolumn{3}{|l|}{ Female (REF) } \\
\hline Male & $0.885(0.8 ; 0.98)$ & 0.0187 \\
\hline \multicolumn{3}{|l|}{ Age group category, years } \\
\hline $35-44$ & 0.977 (0.825; I.157) & 0.7885 \\
\hline $45-54$ & 1.051 (0.898; I.23) & 0.5318 \\
\hline $55-64$ & 1.045 (0.89।; I.227) & 0.5866 \\
\hline $65-74$ & I.I36 (0.93I; I.386) & 0.2086 \\
\hline$\geq 75$ & I.34 (I.037; I.733) & 0.0255 \\
\hline \multicolumn{3}{|l|}{ Region of residence } \\
\hline \multicolumn{3}{|l|}{ Northeast (REF) } \\
\hline Midwest & $1.030(0.886 ; 1.197)$ & 0.7005 \\
\hline South & I.I3I (0.989; I.293) & 0.0722 \\
\hline West & $1.054(0.894 ; 1.242)$ & $0.534 I$ \\
\hline \multicolumn{3}{|l|}{ Race/ethnicity } \\
\hline \multicolumn{3}{|l|}{ Caucasian (REF) } \\
\hline African American & 1.005 (0.886; I.139) & 0.9409 \\
\hline Asian & $0.799(0.505 ; 1.265)$ & 0.3394 \\
\hline Hispanic & $0.850(0.545 ; 1.327)$ & 0.4755 \\
\hline Others/unknown & $0.865(0.747 ; 1.000)$ & 0.0500 \\
\hline \multicolumn{3}{|l|}{ Comorbidity } \\
\hline Major depressive disorder & $0.984(0.858 ; 1.128)$ & 0.8151 \\
\hline Anxiety disorders & $0.996(0.879 ; 1.13)$ & 0.9556 \\
\hline Substance abuse & 1.211 (1.093; I.342) & 0.0003 \\
\hline Hypertension & $1.037(0.922 ; 1.166)$ & 0.5422 \\
\hline Hyperlipidaemia & $0.928(0.825 ; 1.045)$ & 0.217 \\
\hline Diabetes (type I or 2) & I.I3 (0.996; I.282) & 0.0576 \\
\hline
\end{tabular}

(Continued)
Table 4 (Continued).

\begin{tabular}{|l|l|l|}
\hline Parameter & HR (95\% Cl) & p-value $^{\text {a }}$ \\
\hline \multicolumn{1}{|c|}{ Hyperprolactinaemia } & $0.448(0.144 ; 1.393)$ & 0.1652 \\
Insomnia & $0.966(0.809 ; 1.153)$ & 0.7009 \\
\hline $\begin{array}{l}\text { Weigh at index date or within } \\
\text { I month before index date }\end{array}$ & $1.004(1.002 ; 1.007)$ & $<0.000$ I \\
\hline
\end{tabular}

Notes: ${ }^{a}$ Values in bold indicate parameters with significantly increased risk of clinically relevant $(\geq 7 \%)$ weight loss.

Abbreviations: $\mathrm{Cl}$, confidence interval; FGA, first-generation antipsychotic; $H R$, hazard ratio; REF, reference; SGA, second-generation antipsychotic.

behaviour, unhealthy eating, hyperlipidaemia and smoking. ${ }^{9,35,36}$ The STRIDE trial, which investigated the impact of personalised lifestyle changes on weight and diabetes risk in patients with severe mental illness treated with antipsychotics, documented that weight loss is possible in these patients, and has a favourable impact on fasting glucose levels. ${ }^{36}$ Similarly, a switch from a higher to a lower weight gain risk antipsychotic can positively impact body weight and CVD risk factors. ${ }^{37,38}$

Our comparative analysis confirms the position of lurasidone as an antipsychotic that is associated with a low risk of weight gain, as well as the high risk of weight gain associated with olanzapine previously observed in several large systematic reviews and meta-analyses of randomised clinical trials. $^{12,14,31,33}$ These studies consistently showed that the risk of significant increase in weight was highest with olanzapine and clozapine, followed by quetiapine, risperidone and paliperidone. ${ }^{31,33,39,40}$ At the same time, ziprasidone, lurasidone, aripiprazole and haloperidol (listed in a variable order in the various analyses) were consistently ranked among the antipsychotics with a low risk of causing weight gain. ${ }^{12,14,31,39}$ Most analyses in our comparative study were consistent with this ranking of weight gain risk associated with antipsychotics.

Previous studies have shown that clinically relevant weight gain occurs rapidly during treatment with antipsychotics, with the greatest increases taking place within the first 6 weeks of treatment. ${ }^{9,41,42}$ In addition, young patients, patients with a lower baseline BMI and patients who are naïve to antipsychotic treatment are more prone to experiencing weight gain upon initiation of antipsychotics. ${ }^{40-42}$ Consistent with these observations, our real-world analysis showed that the youngest age group (18-34 years) had a significantly higher risk of clinically relevant weight gain, whether defined as a $\geq 7 \%$ 
weight increase from baseline or a shift to a higher BMI category. Given that mean antipsychotic exposure ranged from 70 to 85 days among the cohorts of our study, the observed weight changes occurred rapidly after antipsychotic initiation, consistent with previous studies. ${ }^{9,40,41}$

Besides its retrospective nature, our study has several limitations. First, the database did not contain any information about lifestyle interventions (eg, diet and exercise) or other measures that patients might have undertaken to lose weight while receiving antipsychotic treatment. Second, we did not have data on changes in lipid and glucose metabolism that are closely related to weight changes. Third, we assumed that patients were receiving antipsychotics based on their prescription order observed in the EMR database, but had no information about patient adherence to the prescribed medications. Fourth, we had no information about antipsychotics prescribed before the baseline period or the proportion of patients who were naïve to antipsychotics. Fifth, we did not have information about non-antipsychotic comedication that could influence body weight. Sixth, the follow-up period of up to 12 months, over which weight and BMI records were extracted from the database, was relatively long compared to the average duration of antipsychotic exposure (maximally 3 months). Seventh, the lurasidone cohort was approximately 5- and 10-fold smaller than cohorts B and $\mathrm{C}$, respectively, which may have affected comparisons between cohorts; however, this problem may have been overcome, in part, by comparisons between lurasidone and individual antipsychotics prescribed to a similar number of patients. Eighth, due to the non-randomized nature of this study, the antipsychotic groups differed regarding baseline variables, but we tried to address this by adjusting analyses for relevant baseline differences. Ninth, the selection of patients with sufficient recorded information on weight and BMI might have biased the composition of the study population towards a higher prevalence of overweight and obese patients who needed closer monitoring of these variables. Tenth, as in all studies with secondary use of data, a risk of information bias derived from incomplete or missing data may be considered. This study was limited to patients with at least one valid weight or BMI data during baseline and follow-up period, which might result in some degree of selection bias; thus, results should be interpreted in the context of this study sample. Finally, our findings describe clinical practice in the US, which may not be generalisable to real-life routine clinical practice settings in other countries. Despite these limitations, we believe that the findings from this analysis of a real-world population of $>15,000$ schizophrenia patients can provide clinically valuable insights to the comparative evidence concerning antipsychotics and their impact on weight.

\section{Conclusions}

In conclusion, the results of this real-world analysis of a large population of adult patients with schizophrenia in the US suggest that lurasidone appears to be associated with less weight gain and more weight loss in the setting of routine clinical care, as previously demonstrated in randomised clinical trials. Compared with many other antipsychotics commonly prescribed in clinical practice, lurasidone has a significantly lower risk of causing clinically relevant weight gain and a higher likelihood of being associated with weight loss. This analysis also showed that the majority of patients with schizophrenia continue to be treated with antipsychotics known to be associated with weight gain and other metabolic alterations, despite the increased awareness of the importance of controlling overweight and other risk factors for metabolic syndrome. Clinical considerations other than weight control may justify clinician's selection of antipsychotics; further evaluation is warranted to better understand the clinical rationale behind these treatment practices, especially as meta-analyses indicate much smaller differences between antipsychotics for efficacy than adverse effect measures. ${ }^{12,37}$

\section{Ethics Approval and Informed Consent}

This study was conducted in compliance with all applicable laws regarding subject privacy, including the US Health Insurance Portability and Accountability Act and the European General Data Protection Regulation. The data used in this study were de-identified to prevent any identification of subjects and no primary collection of individual human subject data occurred. Moreover, results were presented in aggregate level omitted subject identification. Being so, the study did not require written informed consent from patients or the approval from the institutional review board or an Ethical Committee.

\section{Funding}

This study was sponsored by Angelini Pharma SpA. 


\section{Disclosure}

Ilena Pochiero, Fabrizio Calisti, Alessandro Comandini, Alessandra Del Vecchio, Maria Teresa Rosignoli, and Agnese Cattaneo are employees of Angelini Pharma S.p.A. Isabella Costamagna was an employee of Angelini Pharma S. p.A at the time of study conduct. Chi-Chang Chen, Franca Heiman, Ilaria Peduto, and Hsiu-Ching Chang are employees of IQVIA. IQVIA received grants from Angelini, during the conduct of the study. Sasikiran Nunna was an employee of IQVIA at the time of study conduct. Dr Christoph Correll has been a consultant and/or advisor to or has received honoraria from Acadia, Alkermes, Allergan, Angelini, Aristo, Axsome, Damitsa, Gedeon Richter, IntraCellular Therapies, Janssen/ J\&J, Hikma, Karuna, LB Pharma, Lundbeck, MedAvanteProPhase, MedInCell, Medscape, Merck, Mitsubishi Tanabe Pharma, Mylan, Neurocrine, Noven, Otsuka, Pfizer, Recordati, Rovi, Servier, Sumitomo Dainippon, Sunovion, Supernus, Takeda, Viatris, and Teva. He provided expert testimony for Janssen and Otsuka. He served on a Data Safety Monitoring Board for Lundbeck, Rovi, Supernus, and Teva. He has received grant support from Janssen and Takeda. He is also a stock option holder of LB Pharma.

\section{References}

1. Hany M, Rehman B, Azhar Y, et al. Schizophrenia. [Updated 2020 Dec 8]. In: StatPearls [Internet]. Treasure Island (FL): StatPearls Publishing; 2021. Available from: https://www.ncbi.nlm.nih.gov/ books/NBK539864/. Accessed May 7, 2021.

2. Schizophrenia. Available from: https://www.who.int/news-room/factsheets/detail/schizophrenia. Accessed May 7, 2021.

3. Kahn RS, Sommer IE, Murray RM, et al. Schizophrenia. Nat Rev Dis Primers. 2015;1(1):15067. doi:10.1038/nrdp.2015.67

4. GBD 2017 Disease and Injury Incidence and Prevalence Collaborators. Global, regional, and national incidence, prevalence, and years lived with disability for 354 diseases and injuries for 195 countries and territories, 1990-2017: a systematic analysis for the Global Burden of Disease Study 2017. Lancet. 2018;392 (10159):1789-1858. doi:10.1016/S0140-6736(18)32279-7

5. Correll CU, Solmi M, Veronese N, et al. Prevalence, incidence and mortality from cardiovascular disease in patients with pooled and specific severe mental illness: a large-scale meta-analysis of 3,211,768 patients and 113,383,368 controls. World Psychiatry. 2017;16(2):163-180. doi:10.1002/wps.20420

6. Meyer JM, Davis VG, McEvoy JP, et al. Impact of antipsychotic treatment on nonfasting triglycerides in the CATIE schizophrenia trial phase 1. Schizophr Res. 2008;103(1-3):104-109. doi:10.1016/j. schres.2008.04.023

7. Vancampfort D, Stubbs B, Mitchell AJ, et al. Risk of metabolic syndrome and its components in people with schizophrenia and related psychotic disorders, bipolar disorder and major depressive disorder: a systematic review and meta-analysis. World Psychiatry. 2015;14 (3):339-347. doi:10.1002/wps.20252

8. Meyer JM, Davis VG, Goff DC, et al. Change in metabolic syndrome parameters with antipsychotic treatment in the CATIE schizophrenia trial: prospective data from phase 1. Schizophr Res. 2008;101(13):273-286. doi:10.1016/j.schres.2007.12.487
9. Carey ME, Barnett J, Doherty Y, et al; On behalf of the STEPWISE Research Group. Reducing weight gain in people with schizophrenia, schizoaffective disorder, and first episode psychosis: describing the process of developing the STructured lifestyle Education for People With SchizophrEnia (STEPWISE) intervention. Pilot Feasibility Stud. 2018;4(1):186. doi:10.1186/s40814-018-0378-1

10. Meyer JM, Ng-Mak DS, Chuang -C-C, Rajagopalan K, Loebel A. Weight changes before and after lurasidone treatment: a real-world analysis using electronic health records. Ann Gen Psychiatry. 2017;16 (1):36. doi:10.1186/s12991-017-0159-x

11. Horacek J, Bubenikova-Valesova V, Kopecek M, et al. Mechanism of action of atypical antipsychotic drugs and the neurobiology of schizophrenia. CNS Drugs. 2006;20(5):389-409. doi:10.2165/ 00023210-200620050-00004

12. Huhn M, Nikolakopoulou A, Schneider-Thoma J, et al. Comparative efficacy and tolerability of 32 oral antipsychotics for the acute treatment of adults with multi-episode schizophrenia: a systematic review and network meta-analysis. Lancet. 2019;394(10202):939-951. doi:10.1016/S0140-6736(19)31135-3

13. Barton BB, Segger F, Fischer K, Obermeier M, Musil R. Update on weight-gain caused by antipsychotics: a systematic review and meta-analysis. Expert Opin Drug Saf. 2020;19(3):295-314. doi:10.1080/14740338.2020.1713091

14. Pillinger T, McCutcheon RA, Vano L, et al. Comparative effects of 18 antipsychotics on metabolic function in patients with schizophrenia, predictors of metabolic dysregulation, and association with psychopathology: a systematic review and network meta-analysis. Lancet Psychiatry. 2020;7(1):64-77. doi:10.1016/S2215-0366(19) 30416-X

15. McIntyre RS. Understanding needs, interactions, treatment, and expectations among individuals affected by bipolar disorder or schizophrenia: the UNITE global survey. J Clin Psychiatry. 2009;70 (suppl 3):05-11. doi:10.4088/JCP.7075su1c.02

16. Tandon R, Lenderking WR, Weiss C, et al. The impact on functioning of second-generation antipsychotic medication side effects for patients with schizophrenia: a worldwide, cross-sectional, web-based survey. Ann Gen Psychiatry. 2020;19(1):42. doi:10.1186/s12991-020-00292-5

17. DiBonaventura M, Gabriel S, Dupclay L, Gupta S, Kim E. A patient perspective of the impact of medication side effects on adherence: results of a cross-sectional nationwide survey of patients with schizophrenia. BMC Psychiatry. 2012;12(1):20. doi:10.1186/1471-244X-12-20

18. Velligan DI, Weiden PJ, Sajatovic M, et al. Assessment of adherence problems in patients with serious and persistent mental illness: recommendations from the expert consensus guidelines. $J$ Psychiatr Pract. 2010;16(1):34-45. doi:10.1097/01.pra.0000367776.96012.ca

19. Jeon SW, Kim Y-K. Unresolved issues for utilization of atypical antipsychotics in schizophrenia: antipsychotic polypharmacy and metabolic syndrome. Int $J$ Mol Sci. 2017;18(10):E2174. doi:10.3390/ijms 18102174

20. Pompili M, Giordano G, Luciano $M$, et al. Unmet needs in schizophrenia. CNS Neurol Disord Drug Targets. 2017;16 (8):870-884. doi:10.2174/1871527316666170803143927

21. Josiassen RC, Joseph A, Kohegyi E, et al. Clozapine augmented with risperidone in the treatment of schizophrenia: a randomized, double-blind, placebo-controlled trial. Am J Psychiatry. 2005;162 (1):130-136. doi:10.1176/appi.ajp.162.1.130

22. US Food and Drug Administration. LATUDA (lurasidone hydrochloride) tablets. Available from: https://www.accessdata.fda.gov/drug satfda docs/label/2013/2006031bls10s11.pdf. Accessed May 7, 2021.

23. latuda-epar-product-information_en.pdf. Available from: https:// www.ema.europa.eu/en/documents/product-information/latuda-eparproduct-information_en.pdf. Accessed May 6, 2021.

24. chmp-post-authorisation-summary-positive-opinion-latuda-ii-29_en. pdf. Available from: https://www.ema.europa.eu/en/documents/smop/ chmp-post-authorisation-summary-positive-opinion-latuda-ii-29_en. pdf. Accessed May 7, 2021. 
25. Loebel A, Cucchiaro J, Sarma K, et al. Efficacy and safety of lurasidone $80 \mathrm{mg} /$ day and $160 \mathrm{mg}$ /day in the treatment of schizophrenia: a randomized, double-blind, placebo- and active-controlled trial. Schizophr Res. 2013;145(1-3):101-109. doi:10.1016/j.schres.20 13.01 .009

26. Loebel A, Cucchiaro J, Xu J, Sarma K, Pikalov A, Kane JM. Effectiveness of lurasidone vs. quetiapine XR for relapse prevention in schizophrenia: a 12-month, double-blind, noninferiority study. Schizophr Res. 2013;147 (1):95-102. doi:10.1016/j.schres.2013.03.013

27. Meltzer HY, Cucchiaro J, Silva R, et al. Lurasidone in the treatment of schizophrenia: a randomized, double-blind, placebo- and Olanzapine-Controlled Study. Am J Psychiatry. 2011;168(9): 957-967. doi:10.1176/appi.ajp.2011.10060907

28. Stahl SM, Cucchiaro J, Simonelli D, Hsu J, Pikalov A, Loebel A. Effectiveness of lurasidone for patients with schizophrenia following 6 weeks of acute treatment with lurasidone, olanzapine, or placebo: a 6-Month, Open-Label, Extension Study. J Clin Psychiatry. 2013;74 (05):507-515. doi:10.4088/JCP.12m08084

29. Meyer JM, Mao Y, Pikalov A, Cucchiaro J, Loebel A. Weight change during long-term treatment with lurasidone: pooled analysis of studies in patients with schizophrenia. Int Clin Psychopharmacol. 2015;30(6):342-350. doi:10.1097/YIC.0000000000000091

30. De Hert M, Detraux J, van Winkel R, Yu W, Correll CU. Metabolic and cardiovascular adverse effects associated with antipsychotic drugs. Nat Rev Endocrinol. 2012;8(2):114-126. doi:10.1038/nrendo.2011.156

31. Leucht S, Cipriani A, Spineli L, et al. Comparative efficacy and tolerability of 15 antipsychotic drugs in schizophrenia: a multiple-treatments meta-analysis. Lancet. 2013;382(9896):95 1-962. doi:10.1016/S0140-6736(13)60733-3

32. Rummel-Kluge C, Komossa K, Schwarz S, et al. Head-to-head comparisons of metabolic side effects of second generation antipsychotics in the treatment of schizophrenia: a systematic review and meta-analysis. Schizophr Res. 2010;123(2-3):225-233. doi:10.1016/j.schres.2010. 07.012

33. Spertus J, Horvitz-Lennon M, Abing H, Normand S-L. Risk of weight gain for specific antipsychotic drugs: a meta-analysis. $\mathrm{Npj}$ Schizophr. 2018;4(1):12. doi:10.1038/s41537-018-0053-9
34. Harvey PD. The clinical utility of lurasidone in schizophrenia: patient considerations. NDT. 2015;1103. doi:10.2147/NDT.S68417

35. Correll CU, Lencz T, Malhotra AK. Antipsychotic drugs and obesity. Trends Mol Med. 2011;17(2):97-107. doi:10.1016/j.molmed.20 10.10 .010

36. Green CA, Yarborough BJH, Leo MC, et al. The STRIDE weight loss and lifestyle intervention for individuals taking antipsychotic medications: a randomized trial. AJP. 2015;172(1):71-81. doi:10.1176/ appi.ajp.2014.14020173

37. Correll CU, Sikich L, Reeves G, et al. Metformin add-on vs. antipsychotic switch vs. continued antipsychotic treatment plus healthy lifestyle education in overweight or obese youth with severe mental illness: results from the IMPACT trial. World Psychiatry. 2020;19 (1):69-80. doi:10.1002/wps.20714

38. Siskind D, Gallagher E, Winckel K, et al. Does switching antipsychotics ameliorate weight gain in patients with severe mental illness? A systematic review and meta-analysis. Schizophr Bull. 2021: sbaa191. doi:10.1093/schbul/sbaa191

39. Dayabandara M, Hanwella R, Ratnatunga S, Seneviratne S, Suraweera C, de Silva V. Antipsychotic-associated weight gain: management strategies and impact on treatment adherence. NDT. 2017;13:2231-2241. doi:10.2147/NDT.S113099

40. Musil R, Obermeier M, Russ P, Hamerle M. Weight gain and antipsychotics: a drug safety review. Expert Opin Drug Saf. 2015;14 (1):73-96. doi:10.1517/14740338.2015.974549

41. Bak M, Fransen A, Janssen J, van Os J, Drukker M. Almost all antipsychotics result in weight gain: a meta-analysis. Zhang XY, ed. PLoS One. 2014;9(4):e94112. doi:10.1371/journal.pone.00 94112

42. De Hert M, Yu W, Detraux J, Sweers K, van Winkel R, Correll CU. Body Weight and Metabolic Adverse Effects of Asenapine, Iloperidone, Lurasidone and Paliperidone in the Treatment of Schizophrenia and Bipolar Disorder: A Systematic Review and Exploratory Meta-Analysis. CNS Drugs. 2012; 2012;26(9):733-7526 (9):733-75. doi:10.2165/11634500-000000000-00000
International Journal of General Medicine

\section{Publish your work in this journal}

The International Journal of General Medicine is an international, peer-reviewed open-access journal that focuses on general and internal medicine, pathogenesis, epidemiology, diagnosis, monitoring and treatment protocols. The journal is characterized by the rapid reporting of reviews, original research and clinical studies across all disease areas. The manuscript management system is completely online and includes a very quick and fair peer-review system, which is all easy to use. Visit http://www.dovepress.com/ testimonials.php to read real quotes from published authors. 